\title{
Linfoma Primário Renal
}

\section{Primary Renal Lymphoma}

Filipa Figueiredo ${ }^{1}$, António P. Matos ${ }^{1,2}$, Orlando Nunes ${ }^{3}$, José Sardinha ${ }^{3}$

Autor Correspondente/Corresponding Author:

António P. Matos [antoniopmatos@gmail.com] Travessa do Castro n³, 1350-070 Lisboa, Portugal

PALAVRAS-CHAVE: Linfoma/diagnóstico por imagem; Neoplasias do Rim/diagnóstico por imagem; Ressonância Magnética

KEYWORDS: Kidney Neoplasms/diagnostic imaging; Lymphoma/diagnostic imaging; Magnetic Resonance Imaging

Mulher de 59 anos, com antecedente conhecido de trombocitémia essencial, medicada com idroxiureia, recorreu ao serviço de urgência por desconforto no flanco esquerdo com cerca de 4 dias de evolução, com alívio em decúbito e sem outros sintomas acompanhantes, nomeadamente urinários. $\bigcirc$ exame objetivo e a avaliação laboratorial revelaram-se normais.

Foi realizada avaliação com tomografia computorizada (TC) e posterior ressonância magnética (RM), revelando lesão sólida centrada no seio renal com $3 \mathrm{~cm}$, com extensão capsular perinéfrica, sem condicionar hidronefrose, invasão vascular nem parenquimatosa, sugerindo linfoma primário renal (LPR) (Fig. 1). Não foram observadas adenopatias retroperitoneais, nem evidência de lesões noutros órgãos. A biópsia da lesão, guiada por TC, veio a confirmar o diagnóstico de linfoma de pequenas células B - zona marginal (Fig. 2). A doente foi submetida a biop- sia óssea/mielograma, cujos resultados foram negativos para linfoma. Iniciou rituximab em monoterapia com boa resposta terapêutica na reavaliação após 6 meses.

O LPR é uma entidade extremamente rara, correspondendo a menos de 1\% das apresentações extraganglionares. ${ }^{1} \bigcirc$ diagnóstico de LPR em RM é sugerido pelo crescimento infiltrativo, com extensão capsular perinéfrica, não invasivo, sinal homogéneo T1/T2, elevada restrição à difusão e características hipovasculares no estudo após contraste. $\mathrm{O}$ acometimento preferencial do seio renal, presente neste caso, coloca como principal diagnóstico diferencial o carcinoma urotelial, no entanto, seria expectável que dadas as suas dimensões, que determinasse hidronefrose e características invasivas mais agressivas (ausente no nosso caso).2,3 A literatura é ainda escassa relativamente ao papel da RM neste contexto. ${ }^{4}$ 

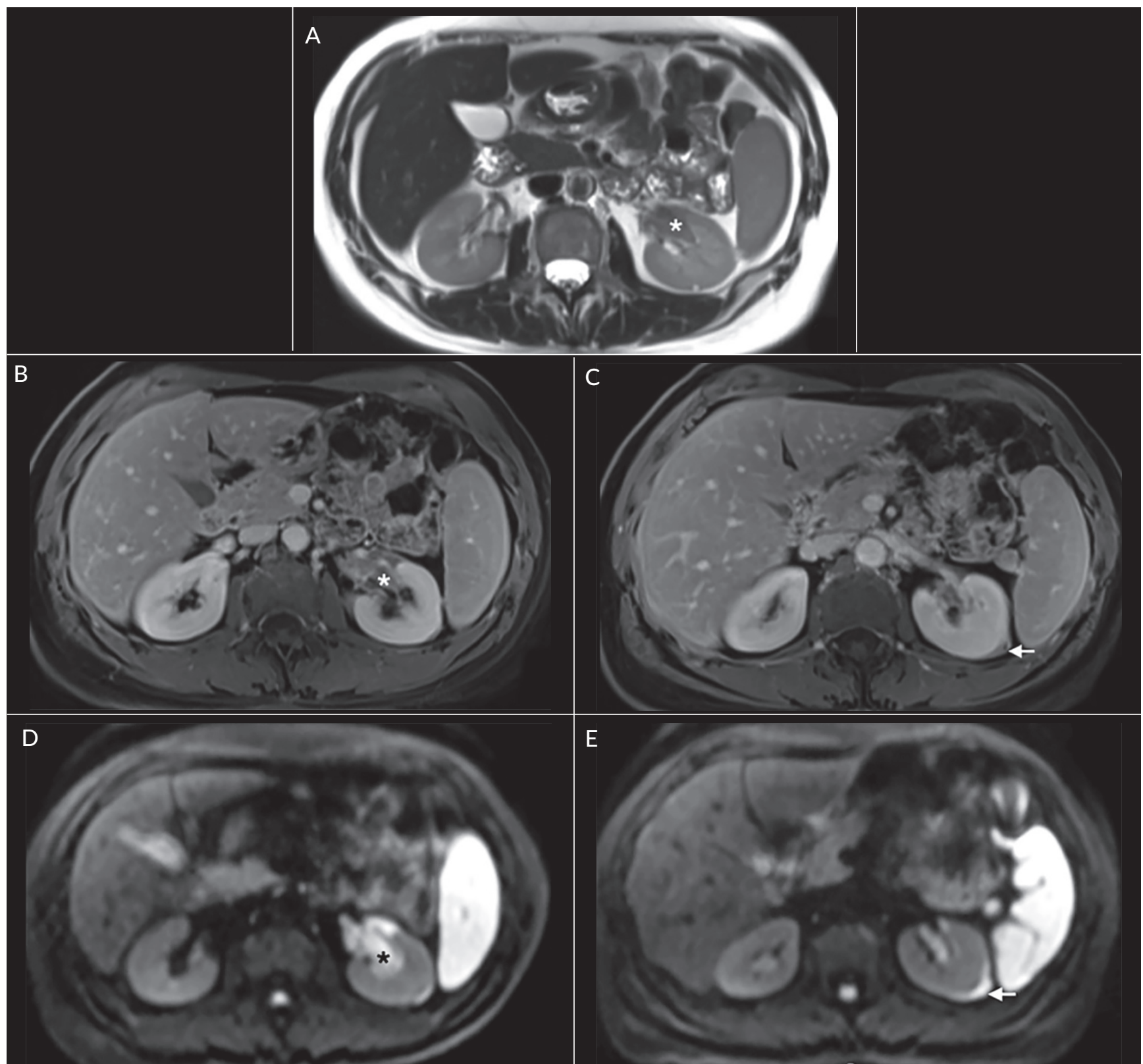

FIGURA 1. Imagens de RM. Axial ponderado em T2 SSFSE (a); axiais em diferentes níveis ponderados em T1-3DGRE após a administração de Gd EV, na fase venosa (b e c); axiais em diferentes níveis ponderados em difusão (DWI) ( $b=1000)$. Nestas imagens observamos uma lesão expansiva centrada ao seio renal ( $\left.{ }^{*}, \mathrm{~A}, \mathrm{~B}, \mathrm{D}\right)$, com $3,8 \mathrm{~cm}$ de maior eixo, sem invasão vascular nem parenquimatosa, encastoando as estruturas hilares. Apesar das suas dimensões, a lesão não determina hidronefrose. Apresenta hipossinal homogéneo T2, marcada restrição à difusão e realce homogéneo na fase venosa. Note-se que a lesão revela extensão capsular perirrenal (seta,C, E). No seu conjunto, estas imagens são sugestivas de linfoma renal.2,5

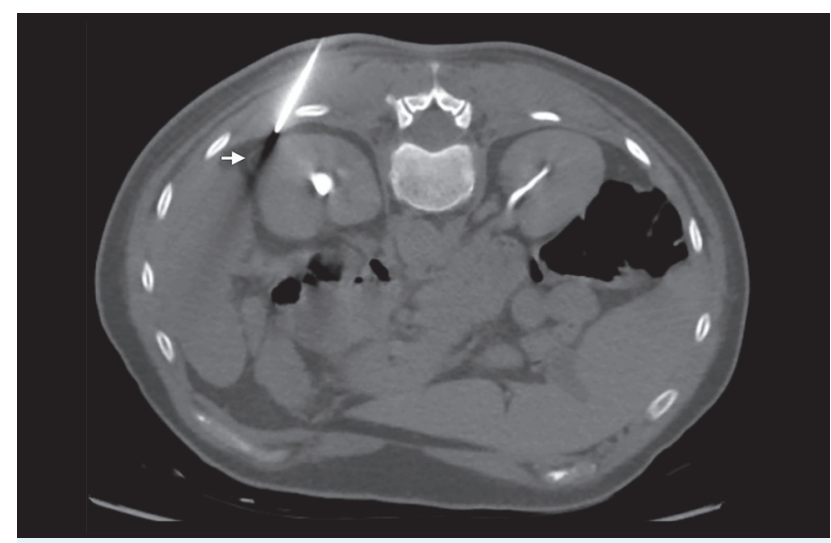

FIGURA 2. Imagem axial de TC, demonstrando uma agulha de biópsia percutânea 18G, para amostragem do espessamento capsular renal descrito e referenciado na RM. O diagnóstico histológico revelou linfoma de pequenas células B - linfoma da zona marginal.
Correto diagnóstico radiológico presuntivo é importante, pois o tratamento e prognóstico do LPR é diferente daquele aplicado às restantes hipóteses diagnósticas, que geralmente englobam a cirurgia como terapêutica curativa definitiva. Os radiologistas têm, assim, um papel fundamental na abordagem a estes doentes, não só na sugestão diagnóstica como na obtenção minimamente invasiva de material histológico.

CONFLITOS DE INTERESSE: Os autores declaram não ter qualquer conflito de interesse na realização do presente trabalho.

FONTES DE FINANCIAMENTO: Não houve qualquer fonte de financiamento na realização do presente trabalho. 
CONFIDENCIALIDADE DOS DADOS: Os autores declaram ter seguido os protocolos da sua instituição acerca da publicação dos dados de doentes.

PROVENIÊNCIA E REVISÃO POR PARES: Não comissionado; revisão externa por pares.

CONFLICTS OF INTEREST: The authors declare that they have no conflicts of interest.

FINANCIAL SUPPORT: This work has not received any contribution, grant or scholarship.

CONFIDENTIALITY OF DATA: The authors declare that they have followed the protocols of their work center on the publication of data from patients.

PROVENANCE AND PEER REVIEW: Not commissioned; externally peer reviewed.

\section{REFERENCIAS}

1. Ganeshan D, Iyer R, Devine C, Bhosale P, Paulson E. Imaging of primary and secondary renal lymphoma. Am J Roentgenol. 2013; 201:W712-9. doi: 10.2214/AJR.13.10669.

2. Purysko AS, Westphalen AC, Remer EM, Coppa CP, Leão FiIho $\mathrm{HM}$, et al. Imaging manifestations of hematologic diseases with renal and perinephric involvement. Radiographics. 2016;36:1038-54. doi: 10.1148/rg.2016150213.

3. Westphalen A, Yeh B, Qayyum A, Hari A, Coakley FV. Differential diagnosis of perinephric masses on CT and MRI. AJR Am J Roentgenol. 2004;183:1697-702.

4. Hauser M, Krestin GP, Hagspiel KD. Bilateral solid multifocal intrarenal and perirenal lesions: differentiation with ultrasonography, computed tomography and magnetic resonance imaging. Clin Radiol. 1995;50:288-94.

5. Zhu Q, Zhu W, Wu J, Chen W. Imaging features of primary renal lymphoma. Acta Radiol. 2018;59:114-20. doi: 10.1177/0284185117706202. 